\title{
Multi-metal additive manufacturing process chain for optical quality mold generation
}

\author{
Biondani, F.G.; Bissacco, G.; Mohanty, S.; Tang, P. T.; Hansen, Hans Nørgaard
}

Published in:

Journal of Materials Processing Technology

Link to article, DOI:

10.1016/j.jmatprotec.2019.116451

Publication date:

2020

Document Version

Peer reviewed version

Link back to DTU Orbit

Citation (APA):

Biondani, F. G., Bissacco, G., Mohanty, S., Tang, P. T., \& Hansen, H. N. (2020). Multi-metal additive manufacturing process chain for optical quality mold generation. Journal of Materials Processing Technology, 277, [116451]. https://doi.org/10.1016/j.jmatprotec.2019.116451

\section{General rights}

Copyright and moral rights for the publications made accessible in the public portal are retained by the authors and/or other copyright owners and it is a condition of accessing publications that users recognise and abide by the legal requirements associated with these rights.

- Users may download and print one copy of any publication from the public portal for the purpose of private study or research.

- You may not further distribute the material or use it for any profit-making activity or commercial gain

- You may freely distribute the URL identifying the publication in the public portal 


\title{
Multi-metal additive manufacturing process chain for optical quality mold generation
}

F. G. Biondani ${ }^{\mathrm{a}, \mathrm{c}}$, G. Bissacco ${ }^{\mathrm{a}}$ S. Mohanty ${ }^{\mathrm{a}}$, P. T. Tang ${ }^{\mathrm{b}}$, Hans Nørgaard Hansen ${ }^{\mathrm{a}}$

${ }^{a}$ Technical University of Denmark, Department of Mechanical Engineering, Produktionstorvet, DK-2800

Kongens Lyngby

${ }^{b}$ IPU, Nils Koppels Alle 404, DK-2800 Kongens Lyngby

'Corresponding author: frgbio@mek.dtu.dk

\begin{abstract}
This paper introduces an innovative process chain for the generation of mold elements based on multimetal additive manufacturing. Starting from an indirect tooling approach for the fabrication of a mold master with optical surface quality, three different additive processes, namely electroforming, thermal spraying and selective laser melting (SLM), are combined to manufacture a test mold element. In order to maintain the targeted optical quality of the mold surface, SLM process parameters optimization has been carried out through numerical simulations. The approach is experimentally verified by generating prototype mold inserts and validated in injection molding trials, showing no signs of degradation of the mold insert after 1000 injection molding cycles.
\end{abstract}

Keywords: Additive Manufacturing, Process chain, Multi-material

\section{Introduction}

In the past decade, metal additive manufacturing (AM) has become an important set of industrial process technologies. The current status of laser based additive manufacturing have been thoroughly discussed by Schmidt et al. (2017), who provided insight on the TRL level of additive manufacturing processes for tooling, aereospace and medical purposes.

The use of metal AM processes is already an established practice in mold making. Petrovic et al. (2011), described the quantitative advantages offered by additive manufacturing for mold making. Specifically, the reduction of the overall mold manufacturing time and the possibility to include conformal cooling channels have so far driven the wide adoption of AM in the mold industry.

For most of the tooling applications (in injection molding), mold post processing, typically performed by mechanical material removal (milling, grinding), is recommended.

When the applications require molds with free form geometries and surface roughness below Sa $30 \mathrm{~nm}$, an additional polishing step must be added to the process chain.

Polishing a 3D geometry while retaining the original shape of the part is indeed a challenging task, that substantially increases mold manufacturing lead time and cost.

Given the complexity of mould 3D shapes, it is common industrial practice to rely on hand polishing of mold surfaces. However, manual polishing lacks repeatability, accuracy and requires highly skilled operators. Manual polishing is a known issue in the mold making industry, and therefore alternative process routes identification is a widely discussed topic. Several researchers have focused on surface post-processes based on electrochemical polishing, controlled mechanical polishing, laser polishing or a combination thereof.

Löber et al. (2013) studied the effects of different post-processing operations on additively manufactured 316L steel parts. Grinding, sand blasting, electrolytic and plasma polishing were compared. By combining mechanical and plasma polishing, a roughness as low as $0.12 \mu \mathrm{m} \mathrm{Ra}$ was achieved, however the uncontrolled material removal of those processes was recognized as a possible issue that needs to be further investigated. 
Pilny and Bissacco (2015), proposed solutions for automated free form polishing based on monitoring strategies for robot assisted polishing with simultaneous analysis of acoustic emission, process forces and scattered light measurements of the part. However, this approach has not yet reached industrial acceptance.

Zhang et al. (2019) developed a conformal polishing tool for linearly extruded geometries. The tool consisting of a PDMS pad with embedded abrasives was used to polish a Selective Laser Melted (SLM) part in 316L stainless steel. As a result, the initial roughness was reduced by $70 \%$ to $5.78 \mu \mathrm{m} \mathrm{Ra}$, which still does not satisfy the requirement for injection molding. Significant form errors were also detecting after the polishing process.

Widlack and Lochynski (2019), analyzed the surface finishing of SLM steel parts after grinding and electropolishing. They concluded that electropolishing alone, although capable of reducing the surface roughness of SLM parts, is not sufficient for achieving high quality surfaces necessary for the food industry. Baicheng et al. (2017) achieved similar results on electropolished Inconel parts: even though electropolishing could reduce the roughness of AM parts from 6 to $3 \mu \mathrm{m}$, the long scale waviness characteristic of polished AM part was still visible.

Beside mechanical and chemical processes, laser polishing has shown great potential in terms of post processing of $3 \mathrm{D}$ geometries.

A detailed review of the physics of the process and applications of laser polishing is given by Krishanan and Fang (2019).

Zhihao et al. (2018) demonstrate the capability of laser polishing by reducing the surface roughness of additively manufactured Inconel parts from $7 \mu \mathrm{m}$ to $0.1 \mu \mathrm{m}$. However, no consideration regarding the preservation of sharp edges was reported. Laser polishing accomplishes surface smoothing by melting the uppermost layer of the workpiece. The redistribution of the molten material under capillary forces evens the surface asperities. However, the smoothing action of the process, makes the preservation of sharp corners and sharp structures problematic.

Optical quality free form surfaces can be obtained by diamond machining as deterministic material removal process thus avoiding variability of the surface quality and lengthy production associated with other polishing processes. Brinksmeier et al. (2002) gave an overview of the capabilities of diamond machining for mold machining applications and metal optics.

The major obstacle for the industrial diffusion of diamond machining is the limited materials choice. The majority of diamond machinable materials do not retain sufficient mechanical properties to guarantee unaffected mold surface quality for long production runs. Diamond machining of steel instead, results in catastrophic wear of the diamond tools. Ultrasonic vibration diamond machining has shown the possibility to significantly reduce diamond tool wear. However, to the authors' knowledge, it has only been developed for turning configuration, while the mold industry substantially relies on ball end milling machining for free form surfaces.

This paper proposes an innovative process chain that delivers durable molds with conformal cooling channels produced by additive manufacturing. The form accuracy and surface finishing is given by a subsequent diamond ball end milling machining process, without the need of any polishing operation.

In order to overcome the known issues in material selection for diamond machining, an indirect manufacturing approach is exploited, involving three different additive processes, namely Selective Laser Melting (SLM), Thermal Spray (TS) and ElectroForming (EF). Process parameters optimization through modelling is applied to SLM in order to thermal relate issues on surface quality and surface hardness of the mold. 


\section{Indirect tooling in molds manufacturing}

Indirect tooling is a broad term that groups all the tooling process chains that shape the mold with the aid of a sacrificial substrate designed to be removed in a subsequent step of the process chains. Hansen et al. (2010), provided a thorough description of the indirect process chains with numerous example of industrial applications. In the case of mold manufacturing, the substrate carries the opposite shape of the final mold that is deposited over the substrate by means of chemical processes such as electroforming. Nickel is commonly used for elecroforming of molds since the characteristic hardness of an electrodeposited nickel coating is in the order of 400-500 HV as reported by Hansen et al. (2010); sufficient for large volume production by injection molding. Given the remarkable replication accuracy of the process, EF finds application in a wide range of engineering fields. Tang (2011), described potential applications of electroforming ranging from MEMS, molds and aerospace industry. Copper or aluminium, which are diamond machinable to nanometric roughness and sub micrometric geometrical accuracy, are preferentially used as substrate materials. On the generated master geometry, a sufficiently thick nickel layer is deposited by EF to generate the mold geometry and, after minor finishing operations, the substrate is removed from the master (typically by selective etching) and the mold is ready to use.

The growth rate of electrodeposited coatings is in the range of 10-100 $\mu \mathrm{m} / \mathrm{h}$, and weeks-long deposition time is often required to produce sufficiently thick layers. The deposition of thick layers comes along with build-up of internal stresses with risk of bending and premature detachment of the tool from the substrate. Furthermore, non-uniformity in the current distribution over the surface results in uneven coating growth that may causes the occurrence of voids in high aspect ratio protruding features. Finally, only full bodied mold elements can be produced in this way, preventing the possibility of inclusion of internal structures such as conformal cooling channels for molding process performance optimization.

\section{Multi-metal additive manufacturing process chain}

The natural combination of metal additive manufacturing with an indirect tooling approach based on EF has a manifold potential. First, it enables the generation of molds with optical surface quality and high accuracy given by diamond machining. Second, it allows the introductions of conformal cooling channels. Further, polishing operations or weeks-long chemical deposition are avoided, consequently lowering the cost and lead time of mold manufacturing. Therefore, in the following study, innovative multi-metal additive technologies are presented as complementary processes to EF.

The proposed process chain exploits the diamond machinability of pure copper to produce a master with optical surface quality and the exact shape of the part to be molded. After diamond machining, nickel is electrodeposited on top of the master and a $500 \mu \mathrm{m}$ thick layer is built, independently from the master size. The purpose of the nickel layer is to transfer the master surface topography on the mold surface while providing sufficient wear resistance for injection molding. The thickness of the electroformed nickel layer needs to be low to avoid excessively long deposition time and irregularity in the layer thickness distribution. On top of the electroformed nickel layer, the full body of the mold must be generated. For this purpose, laser based metal additive processes can be applied.

In this work, validation of the process chain was carried out with respect to a flat mold surface and SLM was chosen as the additive process for the mold body consolidation. The SLM process introduces a considerable heat input, which increases the temperature within the electrodeposited nickel layer, which is characterized by crystal grain size of $10-100 \mathrm{~nm}$. If the recrystallization temperature is reached, the grains will start to coalesce with consequent loss of hardness as reported by (Tang, 2011). Furthermore, 


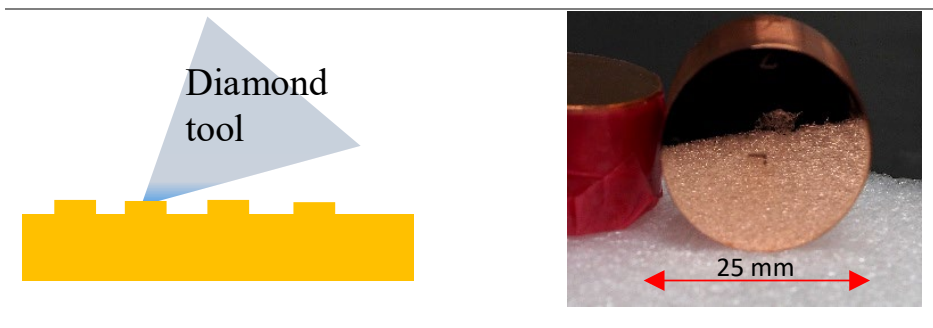

Diamond machining of a copper master ensure geometrical accuracy and nanometric surface roughness

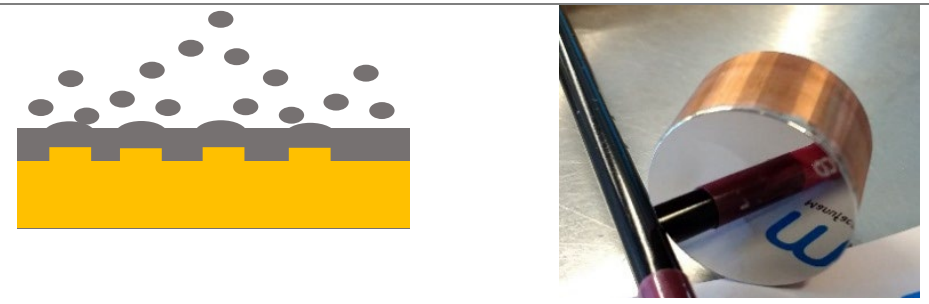

Electroplating of approximately $500 \mu \mathrm{m}$ of Nickel irrespectively from the size of the master, the coating ensures wear resistance while the mechanical strength for handling is given by the subsequent processes

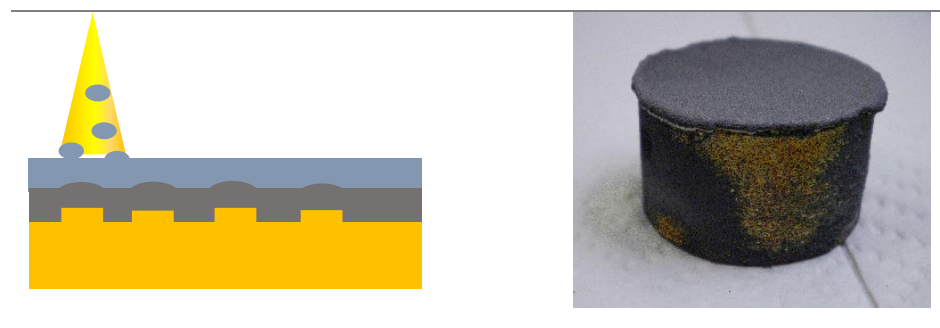

A thermally sprayed metallic buffer layer protect the nickel coating from excessive heat that may led to grain growth

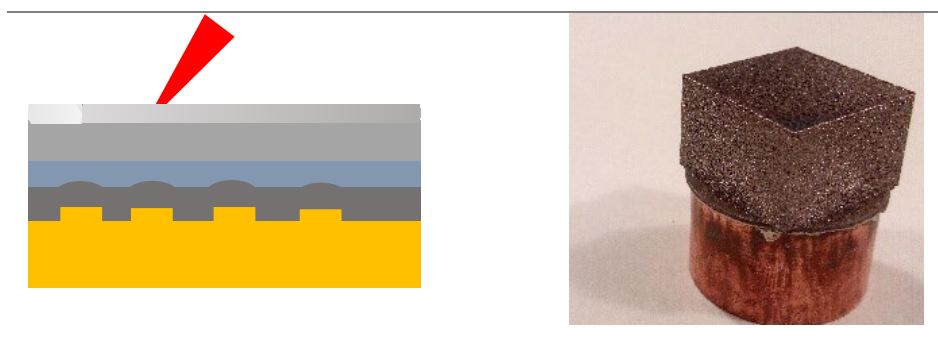

Additive manufacturing of the bulk of the mold ensure mechanical strength, allow to introduce conformal cooling channel and increase the material deposition rate compare of electroforming:

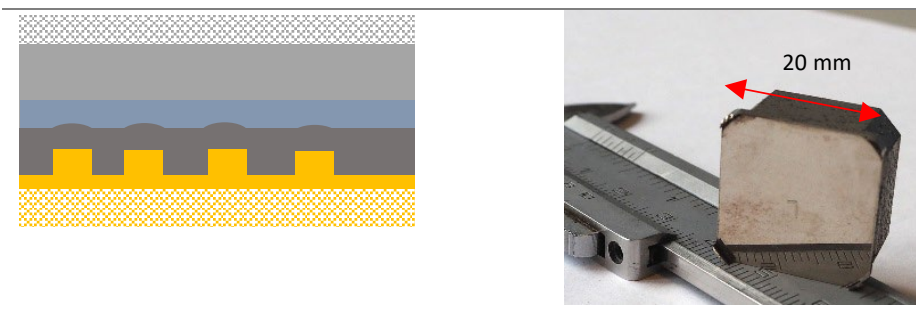

Machining of the reference surfaces and of the bulk of the copper master, followed by chemical removal of the remaining part of the master.
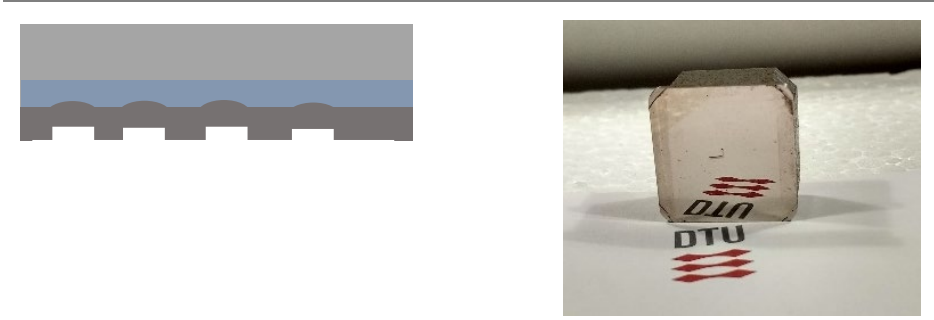

Injection molding using the produced insert

Figure 1: Steps of the proposed process chain. 
the irregular grain growth caused by the recrystallization process will alter the initial surface topography of the nickel layer, with increase of the surface roughness. For electroformed nickel, the recrystallization is triggered by prolonged exposure at temperature above $250{ }^{\circ} \mathrm{C}$. If the combination of time and temperature reached on the nickel surface is such that recrystallization is initiated, the mold element will lose the intended quality.

In order to prevent excessive heating in the electroformed nickel layer, a metal buffer layer is applied by means of thermal spraying. Over the thermally sprayed layer, the mold body is generated in steel by SLM process. Reference surfaces, used for mounting in the mold assembly, are then generated on the mold element by milling. The final step of the process chain is a selective etching process to remove the copper substrate without risk of deterioration of the optical mold surface. The complete process chain is schematically represented in Figure 1.

\subsection{Heat input control of the SLM process}

The successful coupling of the EF process with SLM to generate optical quality molds pivots on a careful control of the temperature in the electroformed nickel layer throughout the entire process chain. In order to contain the temperature field on the surface of the electroformed nickel layer and thus prevent recrystallization, an additional metallic layer is applied between the electroformed nickel and the AM mold body. In this way the melt pool generated by the laser is brought further away from the mold surface. The thermal spraying process is chosen for this step due to the relatively low heat input which is expected to safely keep the temperature below the critical recrystallization value during the deposition of the buffer layer. In thermal spraying, metal particles are partially or completely melted and propelled towards the workpiece, colliding with the surface to be coated and adhering to it. The morphology of such coating shows lamellar structures deriving from the splatting of the metal drops over the surface. The coating builds up from the stacked individual lamellas and adheres to the surface mostly because of mechanical interlocking. The porosity and surface unevenness of thermally sprayed coatings increases with thickness, limiting the applicable thickness of the buffer layer. To increase the adhesion of the coating, the workpiece surface is cleaned and sandblasted. Furthermore the workpiece is preheated to

favor diffusion across the boundary and increase the bond strength. However, in this application, the preheating temperature is limited by the need to avoid recrystallization. To maximize adhesion between the electroformed and thermally sprayed layer and minimize differential thermal expansion during operation, nickel is chosen as material for the buffer layer. Furthermore, the maximum layer thickness is limited to $800 \mu \mathrm{m}$ to avoid excessive porosity. Modelling of the SLM process is used to optimize the process parameters with respect to consolidation, temperature control and minimum thickness of the buffer layer, in order to avoid the critical recrystallization temperature of nickel.

\section{Process modelling \& optimization}

As the recrystallization of nickel layer is a thermally-induced phenomenon, the optimization of SLM parameters to ensure the desired thermal fields is a natural approach. However, in our particular process chain, there is a strong coupling between the thermal spraying procedure and the parameter selection for SLM process. The former process directly determines the thickness of the 'sacrificial' nickel layer, 
thereby influencing not only the geometry of the substrate for SLM but also the level of constraints on the SLM parameters space. Thus, a combined optimization of the thermal spraying process \& SLM is adopted in this study. A thermal model under multi-metal conditions is chosen to simulate the SLM of commercial MS1 maraging steel on the substrate with thermally sprayed nickel layer. The thermal model is based on an equivalent continuum micro-scale model presented by Mohanty and Hattel (2014), and initially developed to predict dimensions of single tracks produced with SLM. In the current implementation, the model not only predicts the temperature fields in the entire sample, but also tracks the area in the two Ni layers subjected to critical recrystallization temperature (set at $250{ }^{0} \mathrm{C}$ ). Further, the model is incorporated into an evolutionary optimization framework (similar to Mohanty and Hattel, 2017) to determine the possible combinations of parameters for the SLM process step.

\subsection{Optimization parameter selection}

In this study, only the power of the laser during SLM is chosen as the adjustable SLM process parameter in order to simplify the process optimization procedure. At a fixed scanning speed value, the power of the laser beam would have a direct influence on the depths and widths of the resultant temperature isotherms. Thus, in order to reduce the risk of recrystallization in EF Ni layer, it would be logical to keep the laser power at a minimum. However, when the hatch distance, i.e. the distance between two successive laser scan lines, is kept constant, the laser power also directly affects the widths of the molten area and thus controls the consolidation in the layer. A minimum target value of $95 \%$ consolidation was chosen for this study and acted as a constraint on the optimization procedure.

In addition to the SLM laser power, the thickness of the thermally sprayed Ni layer was also included as a parameter to be optimized. The primary aim of adding a thermally sprayed layer of $\mathrm{Ni}$, as described before, is to act as a thermal barrier preventing the thermal recrystallization conditions in the electroformed Ni layer. As the adhesion strength and porosity of this thermally sprayed Ni layer worsens with the increase of coating thickness, it is pragmatic to identify its optimum value. This optimum value will be dependent upon the thermal fields during SLM and thus consequently on the chosen process parameters. A hard limit of $800 \mu \mathrm{m}$ is also set as a constraint for the thickness of thermally sprayed layer. The laser scanning speed, hatch spacing and powder-bed layer thickness were set at $0.8 \mathrm{~m} / \mathrm{s}, 100 \mu \mathrm{m}$ and $80 \mu \mathrm{m}$ respectively for an acceptable production time. The waiting time between each layer was greater than $6 \mathrm{~s}$ ensuring that the entire part had a chance to cool down to near ambient temperature conditions.

\subsection{Evolutionary optimization of process chain}

For the current optimization study, two parameters (namely laser power and thermally sprayed layer thickness) are considered with respect to three objectives: thickness of region having critical recrystallization temperature, consolidation in the sample (part density), and thickness of thermally

sprayed Ni layer. The optimization problem is formulated in a multi-objective framework with goals of minimization of the thermally sprayed Ni layer thickness and the thickness of recrystallized region and maximization of the consolidation.

The multi-objective evolutionary algorithm NSGA-II is used for the current study wherein each individual is defined using real number values of the parameters. An initial population size of 100 is chosen, and the optimization is carried out until either 25 generations or until the successive improvement in the population approaches a pre-defined lower limit. The evolutionary algorithm is implemented on six independent runs and the results are merged. 


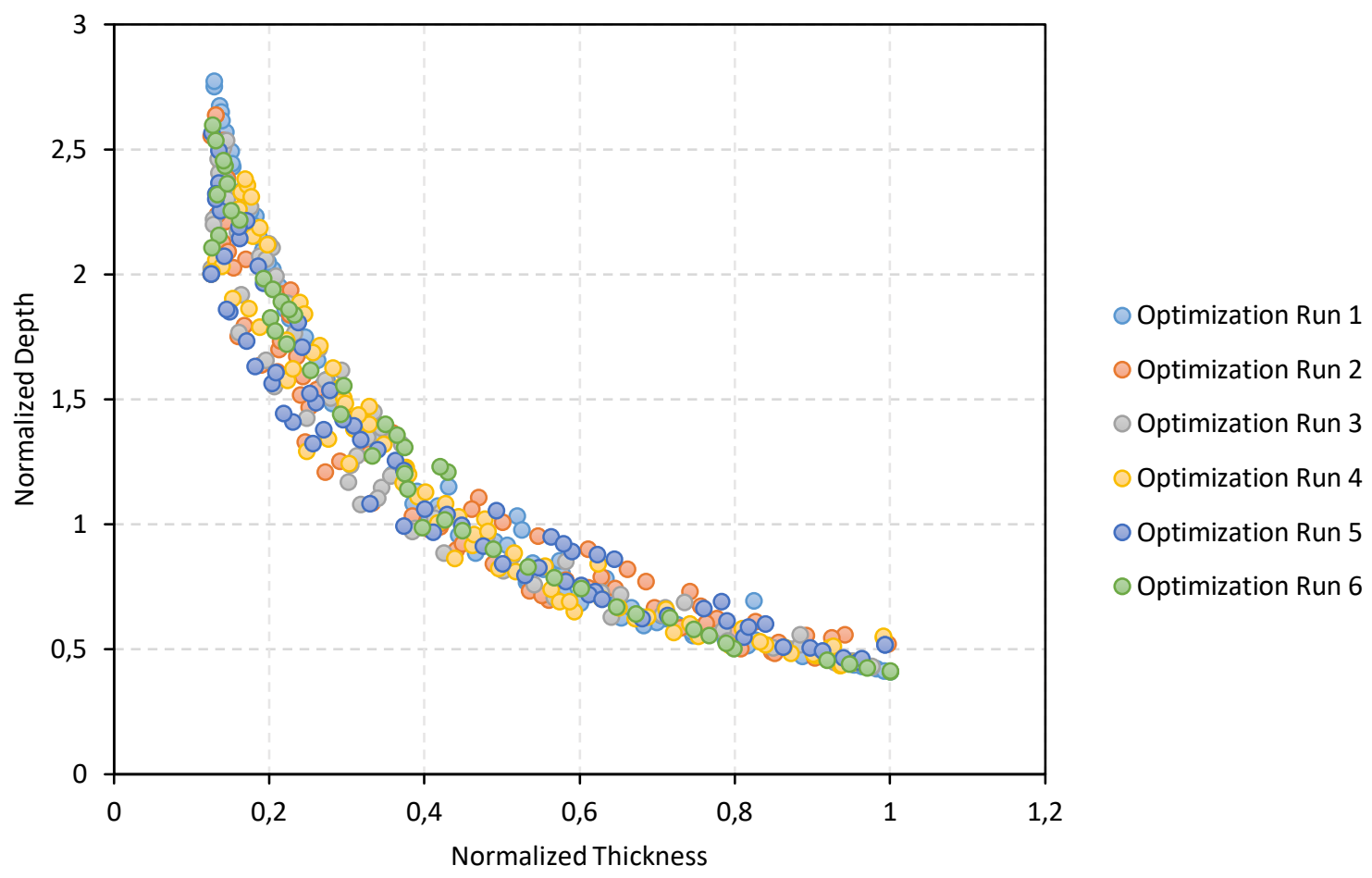

Figure 2: Optimized parameter sets for the process chain collected through six independent optimization runs showing convergence towards a global optimal pareto-front. Note: Each point corresponds to a particular parameter combination.

The optimization procedure resulted in a number of parameter settings from which the combination of $120 \mathrm{~W}$ laser power and $300 \mu \mathrm{m}$ thermal spray layer thickness was chosen from amongst the nondominating solutions. Figure 2 shows the distribution of non-dominating parameter sets on the multiobjective space while Figure 3 shows the corresponding predicted temperature field and regions above critical recrystallization temperature for the chosen parameter set for experiments. In Figure 2, the objective normalized thickness corresponds to the ratio of thermal-spray layer thickness to the maximum layer thickness $(800 \mu \mathrm{m})$, while the objective normalized depth corresponds to the ratio of depth of recrystallized nickel to the corresponding thermal-spray layer thickness. 

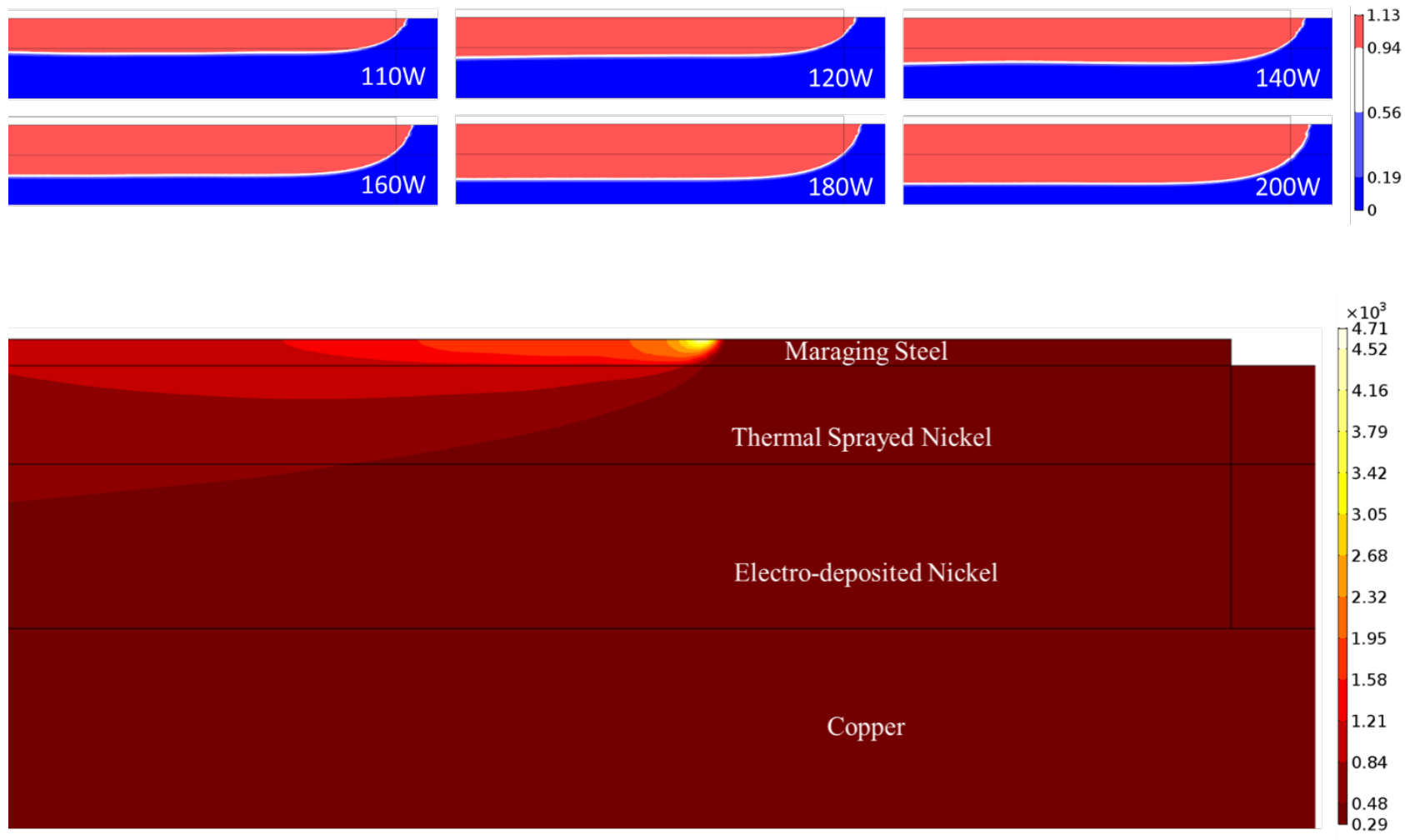

Figure 3: On top: maximum depth of recrystallization in the nickel layers due to different laser powers at $300 \mu \mathrm{m}$ thermal sprayed nickel layer thickness. (Red: recrystallized zone, White: recrystallization threshold Blue: non-recrystallized zone). On the bottom: representative temperature distribution in Kelvin (in the longitudinal plane containing the beam) in the sample during SLM with the chosen optimized process parameters.

\section{Experimental procedure}

To simplify the evaluation of the results, the process chain experimental validation was carried out by the generation of a mold prototype with a flat surface and without cooling channels. A cylinder of CW008A oxygen free copper was chosen as substrate for the EF of nickel. Due to the simple geometry, optical surface quality on the copper surface was obtained by automated polishing in place of diamond machining.

The EF process was carried out in a proprietary nickel sulphamate bath, kept at the constant temperature of $36{ }^{\circ} \mathrm{C}$, and applying a current density of $1.3 \mathrm{~A} / \mathrm{dm}^{2}$. After $30 \mathrm{~h}$ of deposition, a $450 \mu \mathrm{m}$ thick coating was deposited on top of the copper substrate. The metallic buffer layer was deposited on top of the electroformed nickel by means of Atmospheric Plasma Spraying (APS). Pure nickel was chosen for the process in order to ensure continuity with the electroformed layer and a good bonding strength. A 500 $\mu \mathrm{m}$ thick layer was deposited and subsequently machined down to $300 \mu \mathrm{m}$. The temperature of the part during the thermal spraying process was monitored by means of a pyrometer and it did not exceed 175 ${ }^{0} \mathrm{C}$ for the whole deposition time.

The mold body was generated over the thermally sprayed nickel layer by SLM process using an EOSINT M 270 SLM machine and EOS MS1 steel powder (maraging steel with approx. 18\% Ni, 9\% Co, 5\% Mo, 
$0.7 \% \mathrm{Ti}$ ). A full body structure $20 \times 20 \times 12 \mathrm{~mm}$ was produced using the process settings returned by the simulations. After SLM, reference surfaces for mounting were obtained on the back of the mold body by machining, which was also used to reduce the thickness of the copper substrate to reduce selective etching time. Finally the remaining part of the copper substrate was removed by selective etching.

The part was mounted in a standard mold block and used as insert for injection molding trials. 1000 injection molding cycles were carried out using ABS polymer with a melt temperature of $250{ }^{\circ} \mathrm{C}$ and a mold temperature of $60{ }^{\circ} \mathrm{C}$ for the entire duration of the test (approximately six hours). The injection molding parameters are shown in table 1 .

Table 1 Injection molding parameters

\begin{tabular}{lr}
\hline \multicolumn{2}{c}{ Injection molding parameter } \\
\hline Mold temperature & $60{ }^{\circ} \mathrm{C}$ \\
Melt temperature & $250{ }^{\circ} \mathrm{C}$ \\
Injection speed & $125 \mathrm{~mm} / \mathrm{s}$ \\
Holding pressure & $300 \mathrm{bar}$ \\
Cycle time & $21 \mathrm{~s}$ \\
\hline
\end{tabular}

\section{Characterization of the mold insert}

Changes in the surface roughness and significant reduction in hardness represent potential indicators of grain growth in the electroformed nickel layer. Therefore, both Sa surface roughness and HV200g microhardness were monitored throughout the steps of the process chain (Figure 4). The Sa roughness was measured at several stages of the process chain: on the copper sample before EF, on the nickel layer after the removal of the copper master, and on the nickel layer after injection molding. At each step, the values were calculated from the acquisition of four different areas of $250 \times 250 \mu \mathrm{m}$ using a confocal microscope with a 50x magnification lens. No significant changes in the roughness level are observed throughout the process chain. The minor reduction of roughness occurring after the molding trials may be attributed to the removal of residual copper deposited on the nickel surface that was not completely removed via chemical etching.

The hardness value of the EF nickel layer was measured after the removal of the copper master and after injection molding, each averaging six indents. The hardness value of EF nickel "as-deposited" was not measured to avoid damaging the layer. Reference values for identical EF parameter were taken from the work of Tang, (2001).

The comparison of reference values with the hardness measured after SLM and copper dissolution shows an increase in hardness. This increase may be due to internal stresses developed due to the SLM process. No significant variations in the hardness value occurred after injection molding.

XRD stress analysis was carried out on the surface of the EF nickel layer. The results showed compressive stresses of $54+/-10 \mathrm{MPa}$. The observed mild compressive stress state is considered favorable for the application. 

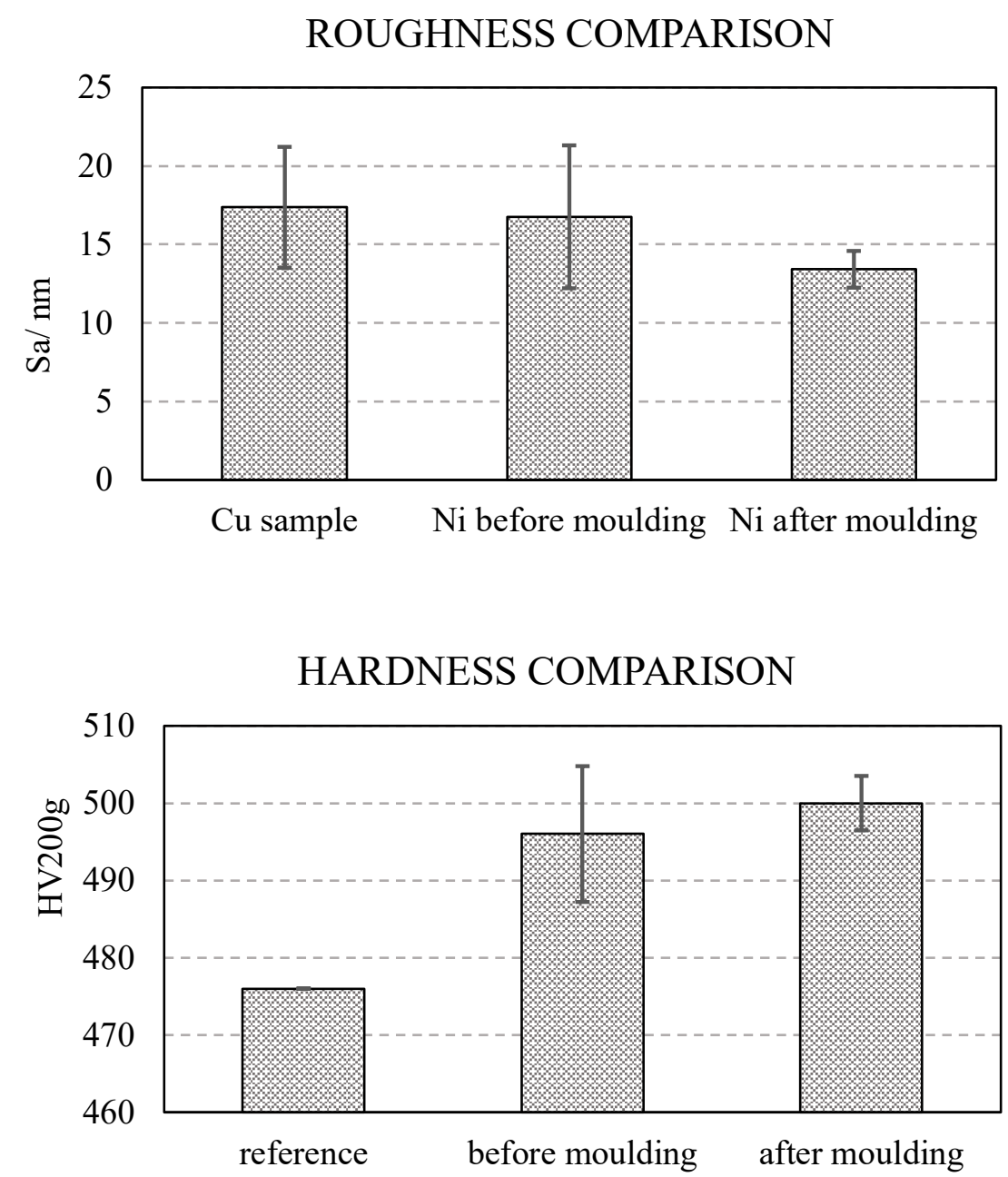

Figure 4: On top: roughness values measured in the different steps of the process chain. On the bottom: comparison of the hardness values of the electroformed Ni layer in the different stages of the process chain. The reference value for "as deposited "Ni was taken from (Tang, 2001). The error bars of both the charts represent $1 \sigma$ standard deviation.

Micrographs of the mold cross section were taken by means of optical microscopy to observe the conditions of the three layers and their interfaces (Figure 5). The thermally sprayed nickel layer contains significant visible porosity. However, the interface between the two nickel layers appears characterized by partial diffusion, increasing the interface strength with respect to mechanical interlocking alone.

\section{Validation through injection molding trials}

During the injection molding process, the insert undergoes a repeated thermal fatigue load. The simultaneous application of heat and pressure can trigger recrystallization in the electroformed nickel as reported by Bissacco et al. (2011), while using nickel molds in an investigation on precision glass molding. Furthermore, internal stresses can arise due to differential thermal expansions of the metal layers of the insert and lead to a potential delamination. 
After 1000 injection cycles, no significant changes in the hardness or in the surface roughness of the tested insert proved the capability of the multi-layered and multi-metal mold to withstand the injection molding process conditions.

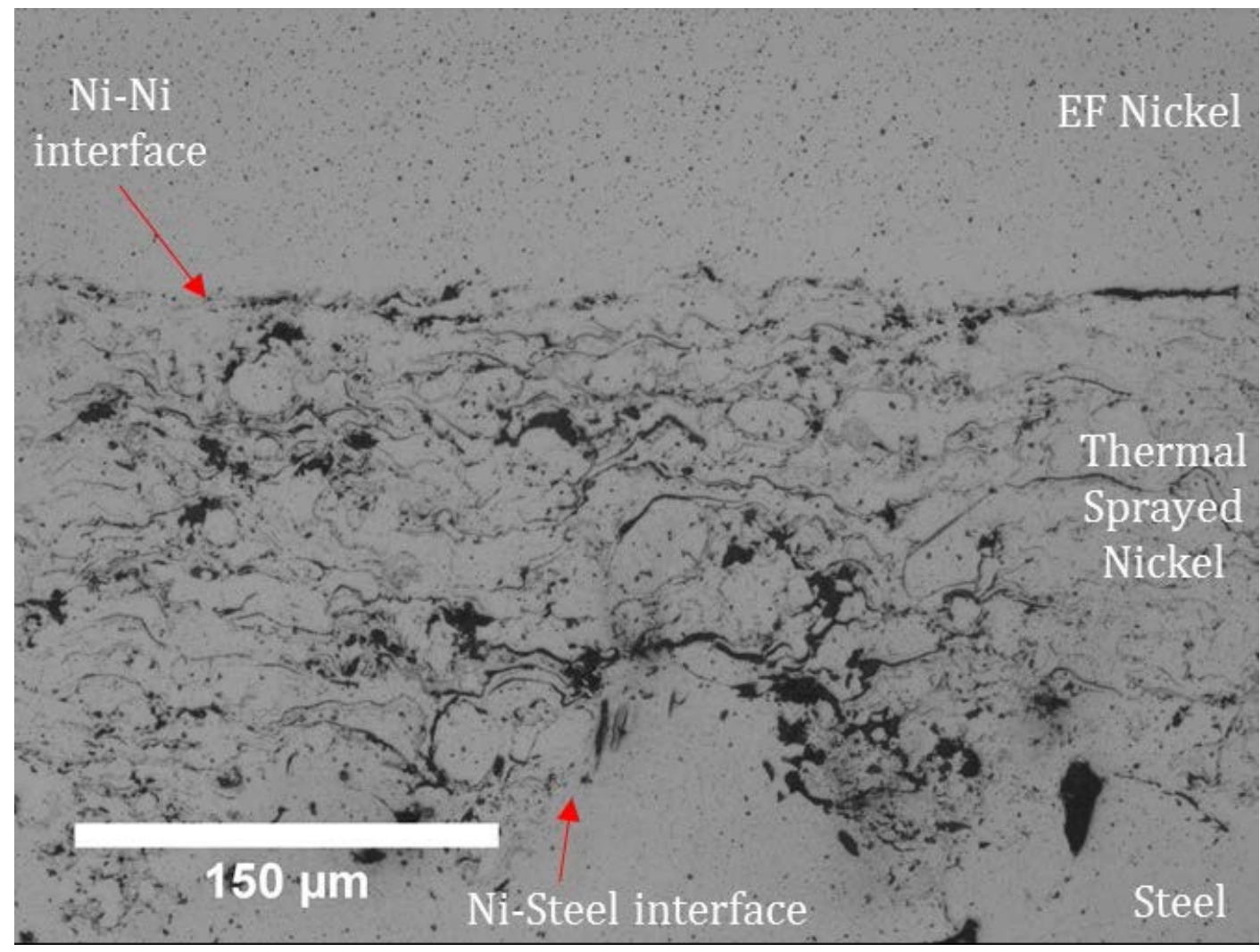

Figure 5 Micrograph of mold cross section showing the three material interfaces.

No delamination occurred between the electroformed layer and the thermally sprayed layer, nor between the thermally sprayed layer and the additively manufactured structure, suggesting that a good interlayer adhesion was achieved.

\section{Conclusions}

In this work, an innovative process chain was proposed for the production of multi-material additively manufactured mold inserts with optical surface quality, exploiting a combination of three different additive processes, namely electroforming (EF), thermal spraying and selective laser melting (SLM). The process chain is suitable for applications where optical surface quality of molds is to be obtained by diamond machining yielding deterministic material removal. The coupling of EF with a higher output additive process such as SLM considerably shortens the EF time, especially for big mold inserts. Furthermore, the SLM process allows realization of features such as conformal cooling channels in the mold body. The proposed process chain was verified by producing a prototype mold insert and validated in injection molding trials, showing no signs of degradation. 


\section{Acknowledgements}

This work was supported by MADE: Manufacturing Academy of Denmark.

\section{References}

Baicheng, Z., Xiaohua, L., Jiaming, B., Junfeng, G., Pan, W., Chen-nan, S., Muiling, N., Guojun, Q., Jun, W., 2017. Study of selective laser melting (SLM) Inconel 718 part surface improvement by electrochemical polishing. Mater. Des. 116, 531-537. https://doi.org/10.1016/j.matdes.2016.11.103

Bissacco, G., Tang, P.T., Hansen., H.N., A., B., Holme, C., 2011. Suitability of Electroformed Nickel Moulds for Wafer Based Precision Glass Moulding, in: Proceedings of the 8th International Conference on MultiMaterial Micro Manufacture. pp. 125-128. https://doi.org/10.3850/978-981-07-0319-6_228

Brinksmeier, E., Gläbe, R., Schönemann, L., 2012. Review on diamond-machining processes for the generation of functional surface structures. CIRP J. Manuf. Sci. Technol. 5, 1-7.

https://doi.org/10.1016/j.cirpj.2011.10.003

Hansen, H.N., Arentoft, M., Tang, P.T., Bissacco, G., Tosello, G., 2010. Tooling process chains and concepts, in: Micro-Manufacturing Engineering and Technology. Elsevier Ltd., pp. 287-297. https://doi.org/10.1016/B978-0-8155-1545-6.00017-X

Krishnan, A., Fang, F., 2019. Review on mechanism and process of surface polishing using lasers. Front. Mech. Eng. 1-21. https://doi.org/10.1007/s11465-019-0535-0

Löber, L., Flache, C., Petters, R., Kühn, U., Eckert, J., 2013. Comparison of different post processing technologies for SLM generated 3161 steel parts. Rapid Prototyp. J. 19, 173-179. https://doi.org/10.1108/13552541311312166

Lyczkowska-Widlak, E., Lochynski, P., Nawrat, G., Chlebus, E., 2019. Comparison of electropolished 316L steel samples manufactured by SLM and traditional technology. Rapid Prototyp. J. 25, 566-580. https://doi.org/10.1108/RPJ-03-2018-0060

Mohanty, S., Hattel, J.H., 2017. Laser additive manufacturing of multimaterial tool inserts: A simulation-based optimization study. Proc. SPIE - Int. Soc. Opt. Eng. 10095, 1-10. https://doi.org/10.1117/12.2253600

Petrovic, V., Vicente Haro Gonzalez, J., Jordá Ferrando, O., Delgado Gordillo, J., Ramón Blasco Puchades, J., Portolés Griñan, L., 2011. Additive layered manufacturing: sectors of industrial application shown through case studies. Int. J. Prod. Res. 49, 1061-1079. https://doi.org/10.1080/00207540903479786

Pilny', L., Bissacco, G., 2015. Development of on the machine process monitoring and control strategy in Robot Assisted Polishing. CIRP Ann. - Manuf. Technol. 64, 313-316. https://doi.org/10.1016/j.cirp.2015.04.013

Schmidt, M., Merklein, M., Bourell, D., Dimitrov, D., Hausotte, T., Wegener, K., Overmeyer, L., Vollertsen, F., Levy, G.N., 2017. Laser based additive manufacturing in industry and academia. CIRP Ann. 66, 561-583. https://doi.org/10.1016/j.cirp.2017.05.011

Tang, P.T., 2011. Electroforming: from Rocket Engines to Nanotweezers. Micro Nanosyst. 3, 180-187.

Tang, P.T., 2001. Pulse reversal plating of nickel and nickel alloys for MEMS, in: Proceedings SUR/FIN. pp. 224-232. https://doi.org/10.1016/S0013-4686(01)00578-3

Zhang, J., Xiang Toh, A.Y., Wang, H., Lu, W.F., Fuh, J.Y.H., 2019. Vibration-assisted conformal polishing of additively manufactured structured surface. Proc. Inst. Mech. Eng. Part C J. Mech. Eng. Sci. 233, 41544164. https://doi.org/10.1177/0954406218811359

Zhihao, F., Libin, L., Longfei, C., Yingchun, G., 2018. Laser Polishing of Additive Manufactured Superalloy. Procedia CIRP 71, 150-154. https://doi.org/10.1016/j.procir.2018.05.088 